\title{
Computing Gutman Connection Index of Thorn Graphs
}

\author{
Muhammad Javaid $\left(\mathbb{D},{ }^{1}\right.$ Muhammad Khubab Siddique, ${ }^{1}$ and Ebenezer Bonyah $\mathbb{D}^{2}$ \\ ${ }^{1}$ Department of Mathematics, School of Science, University of Management and Technology, Lahore 54770, Pakistan \\ ${ }^{2}$ Department of Mathematics Education, Akenten Appiah-Menka University of Skills Training and Entrepreneurial Development, \\ Kumasi 00233, Ghana \\ Correspondence should be addressed to Ebenezer Bonyah; ebbonya@gmail.com
}

Received 17 September 2021; Accepted 27 October 2021; Published 15 November 2021

Academic Editor: Muhammad Imran

Copyright (c) 2021 Muhammad Javaid et al. This is an open access article distributed under the Creative Commons Attribution License, which permits unrestricted use, distribution, and reproduction in any medium, provided the original work is properly cited.

\begin{abstract}
Chemical structural formula can be represented by chemical graphs in which atoms are considered as vertices and bonds between them are considered as edges. A topological index is a real value that is numerically obtained from a chemical graph to predict its various physical and chemical properties. Thorn graphs are obtained by attaching pendant vertices to the different vertices of a graph under certain conditions. In this paper, a numerical relation between the Gutman connection (GC) index of a graph and its thorn graph is established. Moreover, the obtained result is also illustrated by computing the GC index for the particular families of the thorn graphs such as thorn paths, thorn rods, thorn stars, and thorn rings.
\end{abstract}

\section{Introduction}

Let $\Gamma=(V(\Gamma), E(\Gamma))$ be a simple, finite, and connected graph with vertex set $V(\Gamma)$ and edge set $E(\Gamma) \subseteq V(\Gamma) \times V(\Gamma)$. Let $\Theta$ be a collection of such graphs; then, a topological index (TI) is a function from $\Theta$ to the set of real numbers defined under certain conditions on the vertices and edges of the graphs. Moreover, for $\Gamma_{1}, \Gamma_{2} \in \Theta$, if $\Gamma_{1} \cong \Gamma_{2}$, then $\operatorname{TI}\left(\Gamma_{1}\right)=\operatorname{TI}\left(\Gamma_{2}\right)$. The TIs are one of the graph-theoretic techniques which are widely used to study the different properties of the chemical graphs such as boiling point, melting point, flash point, temperature, pressure, tension, heat of evaporation, heat of formation, partition coefficient, retention times in chromatographic, and density $[1,2]$.

TIs are also used in chemoinformatics which is combination of the three different subjects such as information science, chemistry, and mathematics. In chemoinformatics, on the bases of quantitative structural activity relationship (QSAR) and the qualitative structural property relationship (QSPR), the different chemical properties of a chemical graph are correlated with its structure [3,4]. Gutman and Trinajstic [5] evaluated the total $\pi$-electron energy of the molecular structure by using the sum of square of degree (number of neighborhoods) of vertices of molecular graphs that is known by first Zagreb index nowadays. In the same paper, another descriptor appeared that is called as second Zagreb index. Furtula and Gutman [6] introduced another TI called third Zagreb index, which is also known as a forgotten index. After that, many TIs based on the degrees of vertices were established, see [7]. In 2018, Ali and Trinajstic [8] established a descriptor known as modified first Zagreb connection index. In the same paper, they also presented two more descriptors with the name first and second Zagreb connection indices. Ali et al. [9] introduced modified second and third Zagreb connection indices and compared Zagreb connection indices and modified Zagreb connection indices for $T$-sum graphs. Recently, Javaid et al. [10] defined the Gutman connection (GC) index with the help of connection numbers of a graph. For the various computational results, we refer [11-13].

In 1947, Wiener [14] first time applied a distance-based TI to find the boiling point of paraffin. Now, it is called as the Wiener index. Gutman [15] introduced Schultz index of the second kind (Gutman index) as a type of vertex-valencyweighted sum of the distances between all pairs of vertices in a graph. In 1998, Gutman [16] introduced the idea of thorn graph with many applications in chemical graph theory. Bytautas et al. [17] developed an algorithm to find the mean 
Wiener terminal numbers for some thorny graphs. In 2005, Zhou [18] worked on modified Wiener indices for thorn trees. In 2011, Li [19] computed the Zagreb polynomials for thorny graphs. The study of thorn graphs provides mathematical results that relate numerical values of TIs of plerograms and kenograms. Plerograms are obtained from a molecule by expressing each atom with a vertex, but if the hydrogen atoms are not considered, then corresponding mathematical representation of a molecule is called as kenogram. The relation between the terminal Wiener indices of plerograms and kenograms was discussed in [20]. For more details about thorn graphs, see [21-23].

In this study, we establish a relationship between the Gutman connection index of a simple connected graph and its thorn graph. It is also applied to evaluate the Gutman connection index of thorn paths, thorn rods, thorn rings, and thorn stars. Rest of the paper is organized as follows. Section 2 contains the definitions and key concepts that are used in the remaining part of the paper. In Section 3, main and some related results are proved, and in Section 4, application of the main result is discussed for some thorn graphs.

\section{Preliminaries}

Here, $\Gamma$ is considered as a finite connected graphs without loops and multiples edges, and let $V(\Gamma)=\left\{v_{1}, v_{2}, \ldots, v_{n}\right\}$ be its vertex set for an $n$-vertex simple connected graph $\Gamma$. Consider $H=\left(h_{1}, h_{2}, \ldots, h_{n}\right)$ as an $n$-tuple of nonnegative integers. Since distance between any two vertices of $\Gamma$ is the same in both $\Gamma$ and $\Gamma^{h}$, so we denote distance between vertices $u$ and $v$ with respect to both $\Gamma$ and $\Gamma^{h}$ as $d(u, v)$.

2.1. Related Graphs. In this section, we recall the definition of caterpillar, thorn paths, thorn rods, thorn rings, and thorn stars.

Definition 1 (see [24]). For $i=1,2, \ldots, n$, a thorn graph $\Gamma^{h}$ is constructed by attaching $h_{i}$ pendant vertices to the vertex $v_{i}$ of graph $\Gamma$, where $|V(\Gamma)|=n$. If $V_{i}$ is the set of $h_{i}$ thorns of the vertex $v_{i}$, then $V\left(\Gamma^{h}\right)=V(\Gamma) \cup \cup_{i=1}^{n} V_{i}$. For more explanation, see Figure 1.

Definition 2 (see [24]). A thorn path $P_{n, h, k}$ is a graph formed from a path $P_{n}$ by attaching $k$ neighbors to its terminal vertices and $h$ neighbors to its nonterminal vertices. For more detail, see Figure 2.

Definition 3 (see [24]). A caterpillar $\left(T_{m, n}^{\prime}\right)$ is a thorn path obtained from path $P_{n}$ such that its thorn vertices (other than pendant) are of the same degree $m>2$. It is clear that $P_{n, m-2, m-1}=T_{m, n}$, see Figure 3 .

Definition 4 (see [24]). A thorn $\operatorname{rod} P_{n, m}$ is a graph that is obtained by adding $m-1$ pendant vertices to each terminal vertex of $P_{n}$. It is clear that $P_{n, 2, m}=P_{n, m}$, see Figure 4 .

Definition 5 (see [24]). The thorn star $S_{n, h_{1}, h_{2}, \ldots, h_{n}}$ is obtained from the star $S_{n}$ by attaching $h_{i}$ pendant neighbors to vertex $v_{i}$ for $i=1,2, \ldots, n$. Thorn star $S_{n, h_{1}, h_{2}, \ldots, h_{n}}$ defined here is shown in Figure 5.

Definition 6 (see [24]). If for each vertex of a cycle graph $C_{n}$ and a thorn of length $m-2$ is attached, then it is called thorn ring (denoted by $C_{n, m}$ ). For more details, see Figure 6.

2.2. Chemical Applicability of GC Index. This section covers the definition of Gutman connection (GC) index with its applicability.

Definition 7 (see [15]). The Gutman index of a simple connected graph $\Gamma$ (denoted by Gut $(\Gamma)$ ) is defined as

$$
\operatorname{Gut}(\Gamma)=\sum_{\{u, v\} \subseteq \Gamma} d_{\Gamma}(u) d_{\Gamma}(v) d(u, v) .
$$

In the above definition, Javaid et al. [10] replaced the vertex degree with the connection number and defined a new connection-based index known as the Gutman connective (GC) index as follows.

Definition 8. For a simple and connected graph $\Gamma$, the Gutman connection index is

$$
\mathrm{GC}(\Gamma)=\sum_{\{u, v\} \subseteq \Gamma} \tau\left(\frac{u}{\Gamma}\right) \tau\left(\frac{v}{\Gamma}\right) d(u, v),
$$

where $\tau(u / \Gamma)$ and $\tau(v / \Gamma)$ denote the connection number of vertices $u$ and $v$, respectively, of graph $\Gamma$ and $d(u, v)$ is the distance between vertices $u$ and $v$ in $\Gamma$.

The correlation coefficients between the values of GCI and eleven physicochemical properties of octane isomer boiling point (B. P), heat capacity at constant temperature (C. T), heat capacity at constant pressure (C. P), entropy (S), density (D), mean radius ( $\mathrm{Rm} 2)$, change in heat of vaporization $\left(-\Delta H_{v}\right)$, standard heat of formation $\left(-\Delta H_{f}\right)$, accentric factor (A. F), enthalpy of vaporization (HVAP), and standard enthalpy of vaporization (DHVAP) are shown in Table 1. It is clear that absolute value of correlation coefficient of GCI with S, A. F, HVAP, and DHVAP is above 0.9. Also, the value of its correlation coefficient with $\Delta H_{f}$ is 0.8386 . Consequently, the GC index may be a very useful index in the studies of QSPR and QSAR.

Now, before presenting the most frequent used lemma, we define some important notations as $M_{21}(\Gamma)=\sum_{i=1}^{n}$ $\tau\left(v_{i} / \Gamma\right)$ and $M_{2, j}=\sum_{i=1}^{n} \tau\left(v_{i} / \Gamma\right) d\left(v_{i}, v_{j}\right)$, where $j \in$ $\{1,2,3, \ldots, n\}$.

Lemma 1. Let $\Gamma$ be a $\left\{C_{3}, C_{4}\right\}$-free simple and connected graph with vertex set $V(\Gamma)=\left\{v_{1}, v_{2}, \ldots, v_{n}\right\}$ and edge set $E(\Gamma)$. Then, $M_{21}(\Gamma)=M_{1}(\Gamma)-|E(\Gamma)|$, where $M_{1}(\Gamma)$ is the first Zagreb index.

Proof. As $M_{21}(\Gamma)=\sum_{i=1}^{n} \tau\left(v_{i} / \Gamma\right)=\sum_{i=1}^{n} \sum_{u \in N_{\Gamma}\left(v_{i}\right)}(d(u)-1)$, where $N_{\Gamma}\left(v_{i}\right)$ denotes the neighborhood of $v_{i}$, where $\left|N_{\Gamma}\left(v_{i}\right)\right|=d\left(v_{i}\right)$, for all $v_{i} \in V(\Gamma)$. Now, if $u \in N_{\Gamma}\left(v_{i}\right)$, then $v_{i} \in N_{\Gamma}(u)$. Hence, the number of neighborhoods in which 


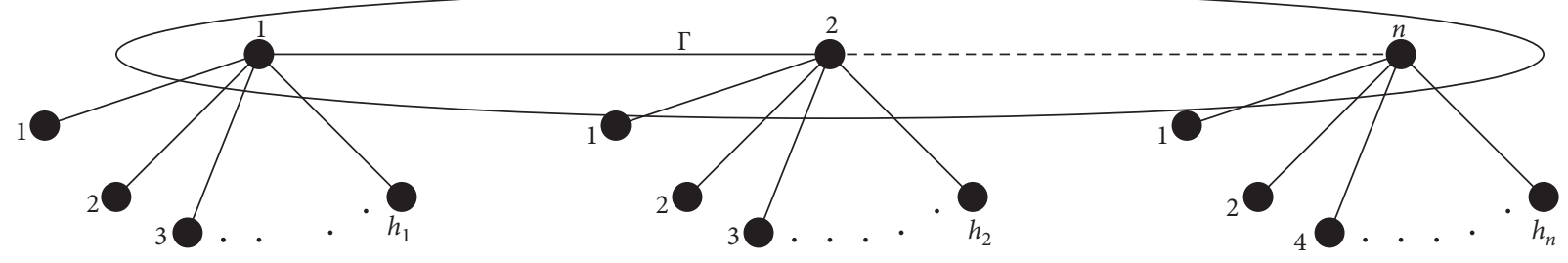

FIgURE 1: Thorn graph $\Gamma^{h}$ of the path graph $\Gamma \cong P_{n}$ with parameter $h=\left(h_{1}, h_{2}, h_{3}, \ldots, h_{n}\right)$.

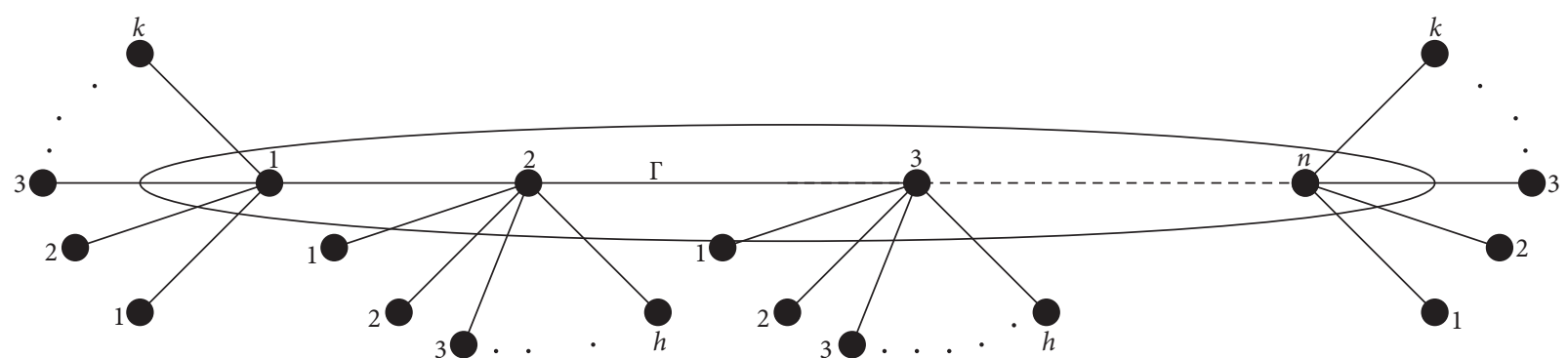

Figure 2: Thorn path, $P_{n, h, k}$.

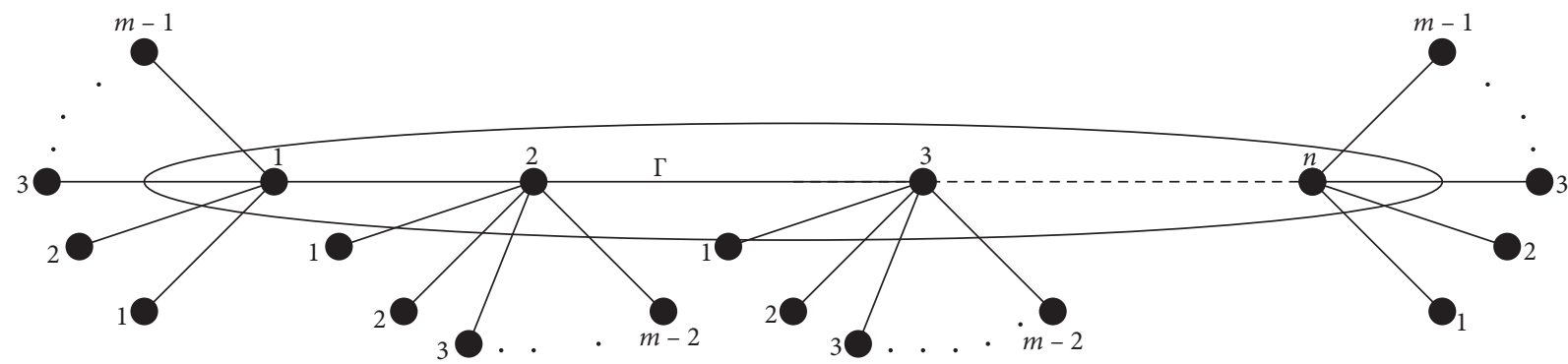

FIgURE 3: Caterpillar, $T_{m, n}{ }^{\prime}$.

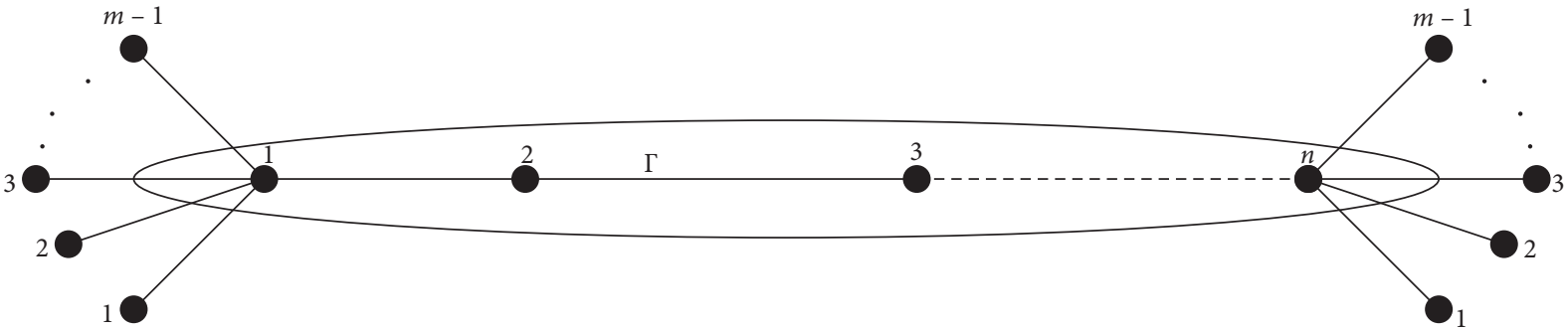

Figure 4: Thorn rod, $P_{n, m}$.

$v_{i}$ lies is equal to the $\left|N_{\Gamma}\left(v_{i}\right)\right|=d\left(v_{i}\right)$, and then, the component of $v_{i}$ which contribute to $\tau(u)$ will be $d\left(v_{i}\right)-1$, for any $u \in N_{\Gamma}\left(v_{i}\right)$. Consequently, $M_{21}(\Gamma)=\sum_{i=1}^{n} d\left(v_{i}\right) \quad(d$ $\left.\left(v_{i}\right)-1\right)=\sum_{i=1}^{n} d\left(v_{i}\right)^{2}-\sum_{i=1}^{n} d\left(v_{i}\right)=M_{1}(\Gamma)-|E(\Gamma)|$.

\section{Main Development}

This section covers the main results of the Gutman connection (GC) index of the thorn graphs in its general form. 


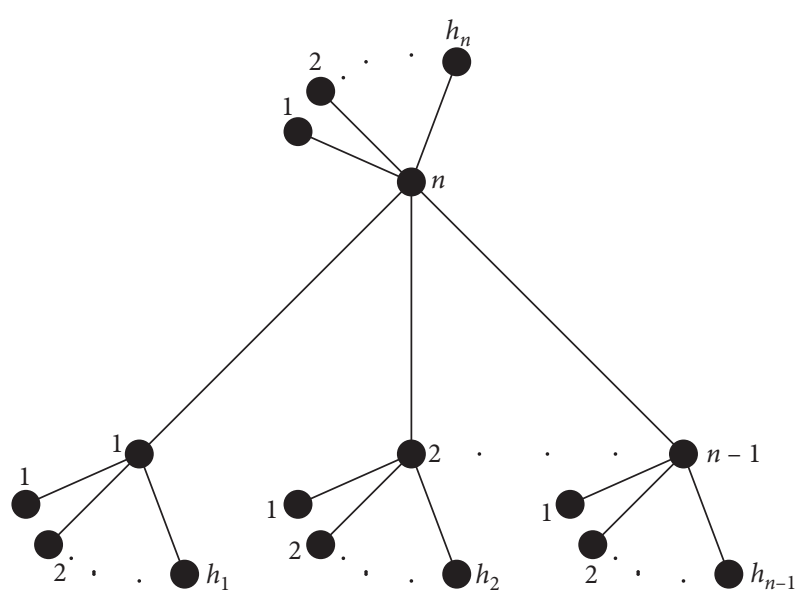

FIgURE 5: Thorn star.

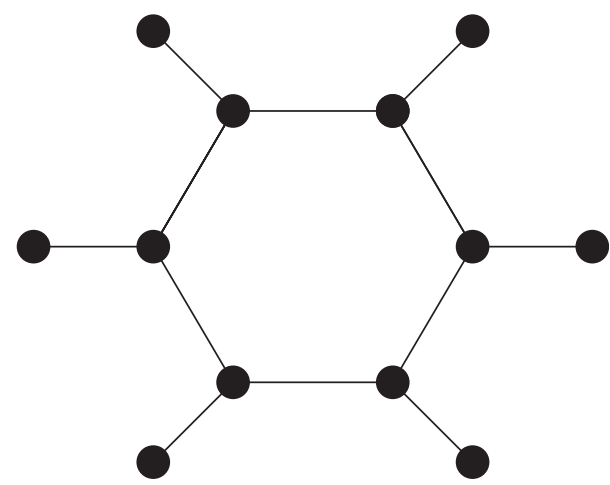

Figure 6: Thorn ring, $C_{5,3}$.

TABle 1: Comparison of correlation coefficients between TIs and physicochemical properties of octane isomers.

\begin{tabular}{lcccccc}
\hline & $\mathrm{GCI}$ & $\mathrm{ZC}_{1}^{*}$ & $\mathrm{M}_{1}$ & $\mathrm{M}_{2}$ & $\mathrm{ZC}_{1}$ & $\mathrm{ZC}_{2}$ \\
\hline B. P & -0.789 & -0.352 & -0.718 & -0.498 & -0.770 & -0.168 \\
C. T & -0.513 & 0.0822 & -0.340 & -0.072 & -0.389 & 0.165 \\
C. P & 0.090 & 0.581 & 0.294 & -0.492 & 0.267 & 0.524 \\
S & -0.910 & -0.891 & -0.954 & -0.942 & -0.920 & -0.836 \\
D & 0.616 & 0.748 & 0.640 & -0.730 & 0.564 & 0.838 \\
$\mathrm{Rm} 2$ & -0.723 & -0.790 & -0.789 & -0.814 & -0.759 & 0.659 \\
$-\Delta H_{f}$ & 0.839 & 0.392 & 0.760 & 0.541 & 0.784 & 0.259 \\
$-\Delta H_{v}$ & -0.171 & -0.071 & -0.222 & -0.129 & -0.294 & 0.145 \\
A. F & -0.901 & -0.949 & -0.973 & -0.986 & -0.951 & -0.862 \\
HVAP & -0.910 & -0.606 & -0.886 & -0.728 & -0.914 & -0.433 \\
DHVAP & -0.941 & -0.705 & -0.936 & -0.812 & -0.955 & -0.551 \\
\hline
\end{tabular}

Theorem 1. Let $\Gamma^{h}$ be a thorn graph of the graph $\Gamma$, where $|V(\Gamma)|=n$; then,

$$
\begin{aligned}
G C\left(\Gamma^{h}\right)= & G C\left(\frac{\Gamma}{\Gamma^{h}}\right)+\sum_{i=1}^{n}\left(d\left(\frac{v_{i}}{\Gamma}\right)+h_{i}-1\right)^{2} h_{i}\left(h_{i}-1\right) \\
& +\sum_{j=1}^{n}\left(d\left(\frac{v_{i}}{\Gamma}\right)+h_{i}-1\right) h_{j}\left(M_{2, j}(\Gamma)+M_{21}(\Gamma)\right. \\
& \left.+\sum_{i=1}^{n} \sum_{p \in N\left(v_{i}\right)} h_{p}\left(d\left(v_{i}, v_{j}\right)+1\right)\right) \\
& +\sum_{1 \leq i<j \leq n}^{n}\left(d\left(\frac{v_{i}}{\Gamma}\right)+h_{i}-1\right)\left(d\left(\frac{v_{i}}{\Gamma}\right)+h_{i}-1\right) \\
& \cdot\left(d\left(v_{i}, v_{j}\right)+2\right) h_{i} h_{j} .
\end{aligned}
$$

Proof. Assume that $\tau\left(u / \Gamma^{h}\right)$ represents the connection numbers of $u$ in graph $\Gamma^{h}$ and $\tau(u / \Gamma)$ represents the connection number of $u$ in graph $\Gamma$. By the definition of the Gutman connection index, we have

$$
\operatorname{GC}\left(\Gamma^{h}\right)=\sum_{\{u, v\} \subseteq V\left(\Gamma^{h}\right)} \tau\left(\frac{u}{\Gamma^{h}}\right) \tau\left(\frac{v}{\Gamma^{h}}\right) d(u, v) .
$$

By the definition of $V\left(\Gamma^{h}\right)$, the sum in equation (5) can be partitioned into four sums as

$$
\sum_{\{u, v\} \subseteq V\left(\Gamma^{h}\right)} \tau\left(\frac{u}{\Gamma^{h}}\right) \tau\left(\frac{v}{\Gamma^{h}}\right) d(u, v)=S_{1}+S_{2}+S_{3}+S_{4},
$$

where $S_{1}$ consists of contributions to $\mathrm{GC}\left(\Gamma^{h}\right)$ of pair of vertices from $\Gamma, S_{2}$ consists of pair of vertices from $V_{i}$, for all $1 \leq i \leq n, S_{3}$ is the contribution of pair of vertices one from $u \in V(\Gamma)$ and the other one $v$ is in $V_{i}$, for all $1 \leq i \leq n$, and $S_{4}$ is taken from all the pair of vertices such that one of them $u$ is from $V_{i}$ and other vertex $v$ from $V j$.

Now,

$$
S_{1}=\sum_{\{u, v\} \subseteq V(\Gamma)} \tau\left(\frac{u}{\Gamma^{h}}\right) \tau\left(\frac{v}{\Gamma^{h}}\right) d(u, v)=\mathrm{GC}\left(\frac{\Gamma}{\Gamma^{h}}\right)
$$

and

$$
\begin{aligned}
S_{2} & =\sum_{i=1}^{n} \sum_{\{u, v\} \subseteq V_{i}} \tau\left(\frac{u}{\Gamma^{h}}\right) \tau\left(\frac{v}{\Gamma^{h}}\right) d(u, v) \\
& =\sum_{i=1}^{n} \sum_{\{u, v\} \subseteq V_{i}}\left(d\left(\frac{v_{i}}{\Gamma}\right)+h_{i}-1\right)\left(d\left(\frac{v_{i}}{\Gamma}\right)+h_{i}-1\right) \cdot 2 \\
& =\sum_{i=1}^{n}\left(d\left(\frac{v_{i}}{\Gamma}\right)+h_{i}-1\right)^{2} \cdot h_{i}\left(h_{i}-1\right) .
\end{aligned}
$$

Similarly, 


$$
\begin{aligned}
& S_{3}=\sum_{i=1}^{n} \sum_{j=1}^{n} \sum_{u=v_{i}} \sum_{v \in V j} \tau\left(\frac{u}{\Gamma^{h}}\right) \tau\left(\frac{v}{\Gamma^{h}}\right) d(u, v) \\
& =\sum_{i=1}^{n} \sum_{j=1}^{n} \sum_{u=v_{i}} \sum_{v \in V j} \tau\left(\frac{u}{\Gamma^{h}}\right)\left(d\left(\frac{v}{\Gamma}\right)+h_{j}-1\right)\left(d\left(v_{i}, v_{j}\right)+1\right) \\
& =\sum_{i=1}^{n} \tau\left(\frac{v_{i}}{\Gamma^{h}}\right) \sum_{j=1}^{n}\left(d\left(\frac{v_{j}}{\Gamma}\right)+h_{j}-1\right) h_{j}\left(d\left(v_{i}, v_{j}\right)+1\right) \\
& =\sum_{j=1}^{n}\left(d\left(\frac{v_{j}}{\Gamma}\right)+h_{j}-1\right) h_{j} \sum_{i=1}^{n} \tau\left(\frac{v_{i}}{\Gamma^{h}}\right)\left(d\left(v_{i}, v_{j}\right)+1\right) \\
& =\sum_{j=1}^{n}\left(d\left(\frac{v_{j}}{\Gamma}\right)+h_{j}-1\right) h_{j} \sum_{i=1}^{n} \\
& \left(\tau\left(\frac{v_{i}}{\Gamma}\right)+\sum_{k \in N\left(v_{i}\right)} h_{k}\right)\left(d\left(v_{i}, v_{j}\right)+1\right) \\
& =\sum_{j=1}^{n}\left(d\left(\frac{v_{j}}{\Gamma}\right)+h_{j}-1\right) h_{j} \\
& \cdot\left(M_{2, j}(\Gamma)+M_{21}(\Gamma)+\sum_{i=1}^{n} \sum_{p \in N\left(v_{i}\right)} h_{p}\left(d\left(v_{i}, v_{j}\right)+1\right)\right),
\end{aligned}
$$

$$
\begin{aligned}
S_{4}= & \sum_{1 \leq i<j \leq n}^{n} \sum_{u \in V_{i}} \sum_{v \in V j} \tau\left(\frac{u}{\Gamma^{h}}\right) \tau\left(\frac{v}{\Gamma^{h}}\right) d(u, v) \\
= & \sum_{1 \leq i<j \leq n}^{n} \sum_{u \in V_{i}} \sum_{v \in V j}\left(d\left(\frac{v_{i}}{\Gamma}\right)+h_{i}-1\right) \\
& \cdot\left(d\left(\frac{v_{j}}{\Gamma}\right)+h_{j}-1\right)\left(d\left(v_{i}, v_{j}\right)+2\right) \\
= & \sum_{1 \leq i<j \leq n}^{n}\left(d\left(\frac{v_{i}}{\Gamma}\right)+h_{i}-1\right)\left(d\left(\frac{v_{j}}{\Gamma}\right)+h_{j}-1\right) \\
& \cdot\left(d\left(v_{i}, v_{j}\right)+2\right)\left(\sum_{u \in V_{i}} 1\right)\left(\sum_{v \in V j} 1\right) \\
= & \sum_{1 \leq i<j \leq n}^{n}\left(d\left(\frac{v_{i}}{\Gamma}\right)+h_{i}-1\right)\left(d\left(\frac{v_{j}}{\Gamma}\right)+h_{j}-1\right)\left(d\left(v_{i}, v_{j}\right)+2\right) h_{i} h_{j} .
\end{aligned}
$$

By substituting the values of $S_{1}, S_{2}, S_{3}$, and $S_{4}$ in equation (5), the required result is obtained.

Now, using Lemma 1 and the result of Theorem 1, we obtain Corollary 1 under the condition on $\Gamma$ that it is free from cycles of length three and four. Moreover, Corollary 2 is obtained by attaching the same number of pendant vertices to each vertex of $\Gamma$.

Corollary 1. If $\Gamma$ is a graph free from cycles of length three and four, then

$$
\begin{aligned}
\mathrm{GC}\left(\Gamma^{h}\right)= & \mathrm{GC}\left(\frac{\Gamma}{\Gamma^{h}}\right)+\sum_{i=1}^{n}\left(d\left(\frac{v_{i}}{\Gamma}\right)+h_{i}-1\right)^{2} \cdot h_{i}\left(h_{i}-1\right)+\sum_{j=1}^{n}\left(d\left(\frac{v_{j}}{\Gamma}\right)+h_{j}-1\right) h_{j} \\
& \cdot\left(M_{2, j}(\Gamma)+M_{1}(\Gamma)-|E(\Gamma)|+\sum_{i=1}^{n} \sum_{p \in N\left(v_{i}\right)} h_{p}\left(d\left(v_{i}, v_{j}\right)+1\right)\right) \\
& +\sum_{1 \leq i<j \leq n}^{n}\left(d\left(\frac{v_{i}}{\Gamma}\right)+h_{i}-1\right)\left(d\left(\frac{v_{j}}{\Gamma}\right)+h_{j}-1\right)\left(d\left(v_{i}, v_{j}\right)+2\right) h_{i} h_{j} .
\end{aligned}
$$

Corollary 2. Let $\Gamma^{h}$ be thorn graph of $\Gamma$ with parameters

$h_{1}=h_{2}=\cdots=h_{n}=h$; then,

$$
\begin{aligned}
\mathrm{GC}\left(\Gamma^{h}\right)= & \mathrm{GC}\left(\frac{\Gamma}{\Gamma^{h}}\right)+h(h-1)\left(M_{1}(\Gamma)+(h-1)^{2} \cdot(n-1)+2(h-1)|E(\Gamma)|\right) \\
& \cdot \sum_{j=1}^{n}\left(d\left(\frac{v_{j}}{\Gamma}\right)+h-1\right) h\left(M_{2, j}(\Gamma)+M_{1}(\Gamma)-|E(\Gamma)|\right)+h \sum_{i=1}^{n} \sum_{p \in N\left(v_{i}\right)}\left(d\left(v_{i}, v_{j}\right)+1\right) \\
& +\sum_{1 \leq i<j \leq n}^{n}\left(d\left(\frac{v_{j}}{\Gamma}\right)+h-1\right)\left(d\left(\frac{v_{j}}{\Gamma}\right)+h-1\right) d\left(\left(v_{i}, v_{j}\right)+2\right) h^{2} .
\end{aligned}
$$




\section{Applications}

In this section, we find the GC index of the thorn path, thorn rod, and thorn ring graphs with the help of the main developed result (Theorem 1).
Theorem 2. Let $n \geq 2$ and $h$ and $k$ be nonnegative integers and $P_{n, h, k}$ be a thorn graph of $P_{n}$; then,

$$
\begin{aligned}
\mathrm{GC}\left(P_{n, h, k}\right)= & \frac{1}{6} h^{4} n^{3}-\frac{13}{6} h^{4} n+3 h^{4}+h^{3} n^{3}-2 h^{3} n^{2}-4 h^{3} n+8 h^{3}+h^{2} k^{2} n^{2}+h^{2} k^{2} n-6 h^{2} k^{2}+h^{2} k n^{2} \\
& -3 h^{2} k n+2 h^{2} k+\frac{13}{6} h^{2} n^{3}-8 h^{2} n^{2}+\frac{59}{6} h^{2} n-7 h^{2}+3 h k^{2} n^{2}-h k^{2} n-10 h k^{2}+3 h k n^{2}-13 h k+16 k n+18 h k+2 h n^{3} \\
& -10 h n^{2}+23 h n-26 h+k^{4} n+3 k^{4}+2 k^{3} n+2 k^{2} n^{2}-k^{2} n-7 k^{2}+2 k n^{2}-10 k n+\frac{2}{3} n^{3}-4 n^{2}+\frac{34}{3} n-14 .
\end{aligned}
$$

Proof. Here, $h_{1}=h_{n}=k$ and $h_{i}=h$, for $2 \leq i \leq n-1$. Now, we find $S_{1}, S_{2}, S_{3}$, and $S_{4}$ as derived in Theorem 1.

$$
\begin{aligned}
& S_{1}=\mathrm{GC}(\Gamma)=\sum_{\{u, v\} \subseteq \Gamma} \tau\left(\frac{u}{\Gamma^{h}}\right) \tau\left(\frac{v}{\Gamma^{h}}\right) d(u, v) \\
& =\tau\left(\frac{v_{1}}{\Gamma^{h}}\right) \sum_{j=3}^{n-2} \tau\left(\frac{v_{j}}{\Gamma^{h}}\right) d\left(v_{1}, v_{j}\right)+\tau\left(\frac{v_{2}}{\Gamma^{h}}\right) \sum_{j=3}^{n-2} \tau\left(\frac{v_{j}}{\Gamma^{h}}\right) d\left(v_{2}, v_{j}\right)+\tau\left(\frac{v_{n-1}}{\Gamma^{h}}\right) \sum_{j=2}^{n-2} \tau\left(\frac{v_{n}}{\Gamma^{h}}\right) d\left(v_{n-1}, v_{j}\right) \\
& +\tau\left(\frac{v_{n}}{\Gamma^{h}}\right) \sum_{j=3}^{n-2} \tau\left(\frac{v_{n}}{\Gamma^{h}}\right) d\left(v_{n}, v_{j}\right)+\tau\left(\frac{v_{1}}{\Gamma^{h}}\right) \tau\left(\frac{v_{2}}{\Gamma^{h}}\right) d\left(v_{1}, v_{2}\right)+\tau\left(\frac{v_{1}}{\Gamma^{h}}\right) \tau\left(\frac{v_{n-1}}{\Gamma^{h}}\right) d\left(v_{1}, v_{n-1}\right) \\
& +\tau\left(\frac{v_{1}}{\Gamma^{h}}\right) \tau\left(\frac{v_{n}}{\Gamma^{h}}\right) d\left(v_{1}, v_{n}\right)+\tau\left(\frac{v_{2}}{\Gamma^{h}}\right) \tau\left(\frac{v_{n-1}}{\Gamma^{h}}\right) d\left(v_{2}, v_{n-1}\right)+\tau\left(\frac{v_{2}}{\Gamma^{h}}\right) \tau\left(\frac{v_{n}}{\Gamma^{h}}\right) d\left(v_{2}, v_{n}\right) \\
& +\tau\left(\frac{v_{n-1}}{\Gamma^{h}}\right) \tau\left(\frac{v_{n}}{\Gamma^{h}}\right) d\left(v_{n-1}, v_{n}\right)+\sum_{\{u, v\} \subseteq V\left(\Gamma /\left\{v_{1}, v_{2}, v_{n-1}, v_{n}\right\}\right)} \tau\left(\frac{u}{\Gamma^{h}}\right) \tau\left(\frac{v}{\Gamma^{h}}\right) d(u, v) \\
& =(h+1) \sum_{j=3}^{n-2}(2 h+2)(j-1)+(h+k+1) \sum_{j=3}^{n-2}(2 h+2)(j-2)+(h+k+1) \sum_{j=3}^{n-2}(2 h+2)(n-1-j) \\
& +(h+1) \sum_{j=3}^{n-2}(2 h+2)(n-j)+(h+1)(h+k+1)+(h+1)(h+k+1)(n-2)+(h+1)^{2}(n-1)+(h+k+1)^{2}(n-3) \\
& +(h+1)(h+k+1)(n-2)+(h+1)(h+k+1)+\sum_{\{u, v\} \subseteq V\left(\Gamma /\left\{v_{1}, v_{2}, v_{n-1}, v_{n}\right\}\right)}(2 h+2) .(2 h+2) d(u, v) \\
& =\sum_{j=3}^{n-2}\left(2(h+1)^{2}(n-1)+2(h+1)(h+k+1)(n-3)\right)+2(h+1)(h+k+1)(n-1)+(h+1)^{2}(n-1) \\
& +(h+k+1)^{2}(n-3)+4(h+1)^{2} \sum_{\{u, v\} \subseteq V\left(\Gamma /\left\{v_{1}, v_{2}, v_{n-1}, v_{n}\right\}\right)} d(u, v)=\left(2(h+1)^{2}(n-1)+2(h+1)(h+k+1)(n-3)\right) \sum_{j=3}^{n-2} 1 \\
& +2(h+1)(h+k+1)(n-1)+(h+1)^{2}(n-1)+(h+k+1)^{2}(n-3)+4(h+1)^{2} \sum_{j=1}^{n-5} \frac{j(j+1)}{2} \\
& =\frac{2}{3} h^{2} n^{3}-4 h^{2} n^{2}+\frac{34}{3} h^{2} n-14 h^{2}+2 h k n^{2}-10 h k n+16 h k+\frac{4}{3} h n^{3}-8 h n^{2}+\frac{68}{3} h n-28 h+k^{2} n-3 k^{2} \\
& +2 k n^{2}-10 k n+16 k+\frac{2}{3} n^{3}-4 n^{2}+\frac{34}{3} n-14 s \text {. }
\end{aligned}
$$


and

$$
\begin{aligned}
S_{2} & =\sum_{i=1}^{n}\left(d\left(\frac{v_{i}}{\Gamma}\right)+h_{i}-1\right)^{2} h_{i}\left(h_{i}-1\right) \\
& =\left(d\left(\frac{v_{1}}{\Gamma}\right)+h_{1}-1\right)^{2} h_{1}\left(h_{1}-1\right)+\sum_{i=2}^{n-1}\left(d\left(\frac{v_{i}}{\Gamma}\right)+h_{i}-1\right)^{2} h_{i}\left(h_{i}-1\right)+\left(d\left(\frac{v_{n}}{\Gamma}\right)+h_{n}-1\right)^{2} h_{n}\left(h_{n}-1\right) \\
& =(1+k-1)^{2} k(k-1)+\sum_{i=2}^{n-1}(2+h-1)^{2} h(h-1)+(1+k-1)^{2} k(k-1) \\
& =2 k^{3}(k-1)+h(h+1)^{2}(h-1)(n-2) .
\end{aligned}
$$

Now, to find $S_{3}, M_{21}$ and $M_{2, j}, j=1,2, \ldots, n$, are required:

$$
\begin{aligned}
M_{21}(\Gamma) & =\sum_{i=1}^{n} \tau\left(v_{i}\right)=1+1+\sum_{i=3}^{n-2} 2+1+1=2+2(n-4)+2=2(n-2)=2 n-4, \\
M_{2,1} & =\sum_{i=1}^{n} \tau\left(\frac{v_{i}}{\Gamma}\right) d\left(v_{i}, v_{1}\right) \\
& =\sum_{i=3}^{n-2} \tau\left(\frac{v_{i}}{\Gamma}\right) d\left(v_{i}, v_{1}\right)+\tau\left(\frac{v_{2}}{\Gamma}\right) d\left(v_{2}, v_{1}\right)+\tau\left(\frac{v_{n-1}}{\Gamma}\right) d\left(v_{n-1}, v_{1}\right)+\tau\left(\frac{v_{n}}{\Gamma}\right) d\left(v_{n}, v_{1}\right) \\
& =2 \sum_{i=3}^{n-2} d\left(v_{i}, v_{1}\right)+1+(n-2)+n-1=(n-2)(n-1)=n^{2}-3 n+2 \\
M_{2,2} & =\sum_{i=1}^{n} \tau\left(\frac{v_{i}}{\Gamma}\right) d\left(v_{i}, v_{2}\right) \\
& =\sum_{i=3}^{n-2} \tau\left(\frac{v_{i}}{\Gamma}\right) d\left(v_{i}, v_{2}\right)+\tau\left(\frac{v_{1}}{\Gamma}\right) d\left(v_{1}, v_{2}\right)+\tau\left(\frac{v_{n-1}}{\Gamma}\right) d\left(v_{n-1}, v_{2}\right)+\tau\left(\frac{v_{n}}{\Gamma}\right) d\left(v_{n}, v_{2}\right) \\
& =2 \sum_{i=3}^{n-2} d\left(v_{i}, v_{2}\right)+1+(n-3)+(n-2)=(n-3)(n-4)+2(n-2)=n^{2}-5 n+8
\end{aligned}
$$

and

$$
\begin{aligned}
M_{2,(n-1)} & =\sum_{i=1}^{n} \tau\left(\frac{v_{i}}{\Gamma}\right) d\left(v_{i}, v_{n-1}\right) \\
& =\sum_{i=3}^{n-2} \tau\left(\frac{v_{i}}{\Gamma}\right) d\left(v_{i}, v_{n-1}\right)+\tau\left(\left(\frac{v_{1}}{\Gamma}\right) d\left(v_{1}, v_{n-1}\right)+\tau\left(\frac{v_{2}}{\Gamma}\right) d\left(v_{2}, v_{n-1}\right)+\tau\left(\frac{v_{n}}{\Gamma}\right) d\left(v_{n}, v_{n-1}\right)\right. \\
& =2 \sum_{i=3}^{n-2} d\left(v_{i}, v_{n-1}\right)+(n-2)+(n-3)+1=(n-3)(n-4)+2(n-2)=n^{2}-5 n+8 .
\end{aligned}
$$


Also,

$$
\begin{aligned}
M_{2, n} & =\sum_{i=1}^{n} \tau\left(\frac{v_{i}}{\Gamma}\right) d\left(v_{i}, v_{n}\right) \\
& =\sum_{i=3}^{n-2} \tau\left(\frac{v_{i}}{\Gamma}\right) d\left(v_{i}, v_{n}\right)+\tau\left(\frac{v_{1}}{\Gamma}\right) d\left(v_{1}, v_{n}\right)+\tau\left(\frac{v_{2}}{\Gamma}\right) d\left(v_{2}, v_{n}\right)+\tau\left(\frac{v_{n-1}}{\Gamma}\right) d\left(v_{n-1}, v_{n}\right) \\
& =2 \sum_{i=3}^{n-2} d\left(v_{i}, v_{n}\right)+(n-1)+(n-2)+1=(n-4)(n-1)+2(n-1)=(n-1)(n-2) .
\end{aligned}
$$

For the next result, we will assume $3 \leq j \leq n-2$ :

$$
\begin{aligned}
M_{2, j} & =\sum_{i=1}^{n} \tau\left(\frac{v_{i}}{\Gamma}\right) d\left(v_{i}, v_{j}\right) \\
& =\tau\left(\frac{v_{1}}{\Gamma}\right) d\left(v_{1}, v_{j}\right)+\tau\left(\frac{v_{2}}{\Gamma}\right) d\left(v_{2}, v_{j}\right)+\tau\left(\frac{v_{n-1}}{\Gamma}\right) d\left(v_{n-1}, v_{j}\right)+\tau\left(\frac{v_{n}}{\Gamma}\right) d\left(v_{n}, v_{j}\right)+\sum_{i=3}^{n-2} \tau\left(\frac{v_{i}}{\Gamma}\right) d\left(v_{i}, v_{j}\right) \\
& =(j-1)+(j-2)+(n-1-j)+(n-j)+2\left(\sum_{i=3}^{j-1}(j-i)+\sum_{i=j+1}^{n-2}(i-j)\right)=2 j^{2}-2 j n-2 j+n^{2}-n+4
\end{aligned}
$$

Now, we take $B_{j}=\sum_{i=1}^{n} \sum_{p \in N\left(v_{i}\right)} h_{p}\left(d\left(v_{i}, v_{j}\right)+1\right)$, for $j=1,2, \ldots, n$. We will find out $B_{1}, B_{2}$, and $B_{n-1}$ and a general expression for $B_{j}$ for $j=3,4, \ldots, n-2$. So,

$$
\begin{aligned}
B_{1}= & \sum_{i=1}^{n} \sum_{p \in N\left(v_{i}\right)} h_{p}\left(d\left(v_{i}, v_{1}\right)+1\right) \\
= & \sum_{p \in N\left(v_{1}\right)} h_{p}\left(d\left(v_{1}, v_{1}\right)+1\right)+\sum_{p \in N\left(v_{2}\right)} h_{p}\left(d\left(v_{2}, v_{1}\right)+1\right)+\sum_{p \in N\left(v_{n-1}\right)} h_{p}\left(d\left(v_{n-1}, v_{1}\right)+1\right) \\
& +\sum_{p \in N\left(v_{n}\right)} h_{p}\left(d\left(v_{n}, v_{1}\right)+1\right)+\sum_{i=3}^{n-2} \sum_{p \in N\left(v_{i}\right)} h_{p}\left(d\left(v_{i}, v_{1}\right)+1\right) \\
= & h(1)+(h+k)(2)+(h+k)(n-1)+h(n)+2 h \sum_{i=3}^{n-2}(j-1+1) \\
= & (n+1)(2 h+k)+h(n-4)(n+1)=(n+1)(2 h+k+h(n-4))=(n+1)(k+(n-2) h),
\end{aligned}
$$




$$
\begin{aligned}
B_{2}= & \sum_{i=1}^{n} \sum_{p \in N\left(v_{i}\right)} h_{p}\left(d\left(v_{i}, v_{2}\right)+1\right) \\
= & \sum_{p \in N\left(v_{1}\right)} h_{p}\left(d\left(v_{1}, v_{2}\right)+1\right)+\sum_{p \in N\left(v_{2}\right)} h_{p}\left(d\left(v_{2}, v_{2}\right)+1\right)+\sum_{p \in N\left(v_{n-1}\right)} h_{p}\left(d\left(v_{n-1}, v_{2}\right)+1\right)+\sum_{p \in N\left(v_{n}\right)} h_{p}\left(d\left(v_{n}, v_{2}\right)+1\right) \\
& +\sum_{i=3}^{n-2} \sum_{p \in N\left(v_{i}\right)} h_{p}\left(d\left(v_{i}, v_{2}\right)+1\right)=h(2)+(h+k)(1)+(h+k)(n-2)+h(n-1)+2 h \sum_{i=3}^{n-2}(i-2+1) \\
= & 2 n h+k(n-1)+h(n-4)(n-1) .
\end{aligned}
$$

$$
B_{n-1}=(n+1)(2 h+k)+h(n-4)(n+1) \text { and } B_{n}=2 n h \quad \text { For } 3 \leq j \leq n-2,
$$

$$
+k(n-1)+h(n-4)(n-1) \text {. }
$$

$$
\begin{aligned}
B_{j}= & \sum_{i=1}^{n} \sum_{p \in N\left(v_{i}\right)} h_{p}\left(d\left(v_{i}, v_{j}\right)+1\right) \\
= & \sum_{p \in N\left(v_{1}\right)} h_{p}\left(d\left(v_{1}, v_{j}\right)+1\right)+\sum_{p \in N\left(v_{2}\right)} h_{p}\left(d\left(v_{2}, v_{j}\right)+1\right)+\sum_{p \in N(v n-1)} h_{p}\left(d\left(v_{n-1}, v_{j}\right)+1\right) \\
& +\sum_{p \in N\left(v_{n}\right)} h_{p}\left(d\left(v_{i}, v_{j}\right)+1\right)+\sum_{i=3}^{n-2} \sum_{p \in N\left(v_{i}\right)} h_{p}\left(d\left(v_{i}, v_{j}\right)+1\right) \\
= & h(j-1+1)+(h+k)(j-2+1)+(h+k)(n-1-j+1)+h(n-j+1)+\sum_{i=3}^{n-2}(h+h)\left(d\left(v_{i}, v_{j}\right)+1\right) \\
= & 2 n h+k(n-1)+2 h\left(\sum_{i=3}^{j}\left(d\left(v_{i}, v_{j}\right)+1\right)+\sum_{i=j+1}^{n-2}\left(d\left(v_{i}, v_{j}\right)+1\right)\right) \\
= & 2 n h+k(n-1)+2 h\left(\sum_{i=3}^{j}(j-i+1)+\sum_{i=j+1}^{n-2}(i-j+1)\right) \\
= & 2 n h+k(n-1)+h((j-1)(j-2)+(n-j-2)(n-j+1)) .
\end{aligned}
$$

So,

$$
\begin{aligned}
S_{3}= & \sum_{j=1}^{n}\left(d\left(\frac{v_{j}}{\Gamma}\right) h_{j}-1\right) h_{j}\left(M_{2, j}(\Gamma)+M_{21}(\Gamma)+B_{j}\right) \\
= & \left(d\left(\frac{v_{1}}{\Gamma}\right) h_{1}-1\right) h_{1}\left(M_{2,1}(\Gamma)+M_{21}(\Gamma)+B_{1}\right)+\left(d\left(v_{2} \Gamma\right)+h_{2}-1\right) h_{2}\left(M_{2,2}(\Gamma)+M_{21}(\Gamma)+B_{2}\right) \\
& +\left(d\left(\frac{v_{n-1}}{\Gamma}\right) h_{n-1}-1\right) h_{1}\left(M_{2,(n-1)}(\Gamma)+M_{21}(\Gamma)+B_{n-1}\right)+\left(d\left(\frac{v_{n}}{\Gamma}\right) h_{n}-1\right) h_{n}\left(M_{2, n}(\Gamma)+M_{21}(\Gamma)+B_{n}\right) \\
& +\sum_{j=3}^{n-2}\left(d\left(\frac{v_{j}}{\Gamma}\right) h_{j}-1\right) h_{j}\left(M_{2, j}(\Gamma)+M_{21}(\Gamma)+B_{j}\right) \\
= & (1+k-1) k\left(n^{2}-3 n+2+2 n-4+(n+1)(k+(n-2) h)+(2+h-1) h\right.
\end{aligned}
$$




$$
\begin{aligned}
& \left(n^{2}-5 n+8+2 n-4+2 n h+k(n-1)+h(n-4)(n-1)\right)+(2+h-1) h\left(n^{2}-5 n+8+2 n-4\right) \\
& +h(n-4)(n-1))+(1+k-1) k\left(n^{2}-3 n+2+2 n-4+(n+1)(k+(n-2) h)\right. \\
& +\sum_{j=3}^{n-2}(2+h-1) h\left(M_{2, j}(\Gamma)+2 n-4+2 n h+k(n-1)\right)+h((j-1)(j-2)+(n-j-2)(n-j+1)) \\
= & 2 k^{2}\left(n^{2}-n-2+(n+1)(k+(n-2) h)\right)+2 h(h+1)\left(n^{2}-3 n+4+2 n h+k(n-1)+h(n-4)(n-1)\right) \\
& +h(h+1) \sum_{j=3}^{n-2} 2 j^{2}-2 j n-2 j+n^{2}-n+4+2 n-4+2 n h+k(n-1)+h((j-1)(j-2)+(n-j-2)(n-j+1)) \\
= & 2 k^{2}\left(n^{2}-n-2+(n+1)(k+(n-2) h)\right)+2 h(h+1)\left(n^{2}-3 n+4+2 n h+k(n-1)+h(n-4)(n-1)\right) \\
& +h(h+1)\left(4 k-8 h+\frac{22}{3} n-4 h n^{2}+\frac{2}{3} h n^{3}+k n^{2}+\frac{22}{3} h n-5 k n-4 n^{2}+\frac{2}{3} n^{3}-8\right) .
\end{aligned}
$$

Also,

$$
\begin{aligned}
S_{4}= & \sum_{1 \leq i<j \leq n}\left(d\left(\frac{v_{i}}{\Gamma}\right)+h_{i}-1\right)\left(d\left(\frac{v_{j}}{\Gamma}\right)+h_{j}-1\right)\left(d\left(v_{i}, v_{j}\right)+2\right) h_{i} h_{j} \\
= & \left(d\left(\frac{v_{1}}{\Gamma}\right)+h_{1}-1\right)\left(d\left(\frac{v_{n}}{\Gamma}\right)+h_{n}-1\right)\left(d\left(v_{1}, v_{n}\right)+2\right) h_{1} h_{n}+\sum_{j=2}^{n-1}\left(d\left(\frac{v_{1}}{\Gamma}\right)+h_{1}-1\right)\left(d\left(\frac{v_{j}}{\Gamma}\right)+h_{j}-1\right) \\
& \cdot\left(d\left(v_{1}, v_{j}\right)+2\right) h_{1} h_{j}+\sum_{j=2}^{n-1}\left(d\left(\frac{v_{n}}{\Gamma}\right)+h_{n}-1\right)\left(d\left(\frac{v_{j}}{\Gamma}\right)+h_{j}-1\right)\left(d\left(v_{n}, v_{j}\right)+2\right) h_{n} h_{j} \\
& +\sum_{2 \leq i<j \leq n-1}\left(d\left(\frac{v_{i}}{\Gamma}\right)+h_{i}-1\right)\left(d\left(\frac{v_{j}}{\Gamma}\right)+h_{j}-1\right)\left(d\left(v_{i}, v_{j}\right)+2\right) h_{i} h_{j} \\
= & (1+k-1)(1+k-1)(n+1) k \cdot k+\sum_{j=2}^{n-1}(1+k-1)(2+h-1)(j-1+2) k h \\
& +\sum_{j=2}^{n-1}(1+k-1)(2+h-1)(n-j+2) k h+\sum_{2 \leq i<j \leq n-1}(2+h-1)(2+h-1)(j-i+2) h h \\
= & k^{4}(n+1)+k^{2} h(h+1) \sum_{j=2}^{n-1}(j+1)+k^{2} h(h+1) \sum_{j=2}^{n-1}(n-j+2)+h^{2}(h+1)^{2} \sum_{2 \leq i<j \leq n-1}(j-i+2)
\end{aligned}
$$

By substituting the values in $S_{1}, S_{2}, S_{3}$, and $S_{4}$ in equation (5), we will get the required result.

For $k=m-2$ and $h=m-1$, thorn path $P_{n, h, k}$ represents a caterpillar $T_{m, n}$. Similarly, a thorn path $P_{n, h, k}$ will be thorn $\operatorname{rod} P_{n, m}$ if $h=0$ and $k=m-1$, i.e., $P_{n, m}=P_{n, 0, m-1}$. Thus, the GI index of the thorn path and thorn rod is defined in the following corollaries.

Corollary 3. For $n, m \geq 2$, the GC index of caterpillar is

$$
\begin{aligned}
\mathrm{GC}\left(T^{\prime} m, n\right)= & \frac{1}{6} m^{4} n^{3}+m^{4} n^{2}-\frac{1}{6} m^{4} n+\frac{1}{3} m^{3} n^{3}-4 m^{3} n^{2} \\
& -\frac{34}{3} m^{3} n+\frac{1}{6} m^{2} n^{3} \\
& -3 m^{2} n^{2}+\frac{221}{6} m^{2} n+34 m^{2}+2 m n^{2}-10 m n \\
& -104 m+4 n+36
\end{aligned}
$$


Corollary 4. For $n, m \geq 2$, the GC index of thorn rod is

$$
\begin{aligned}
\mathrm{GC}\left(P_{n, m}\right)= & m^{4} n+3 m^{4}-2 m^{3} n-12 m^{3} \\
& +2 m^{2} n^{2}-m^{2} n+11 m^{2} \\
& -2 m n^{2}-6 m n+18 m+\frac{2}{3} n^{3}-4 n^{2}+\frac{58}{3} n-34 .
\end{aligned}
$$

Now, we present the GC index for the thorn star. Suppose that $L$ is the number of all pendant vertices other than the pendant vertices attached to $n$th vertex, i.e., $L=\sum_{p=1}^{n-1} h_{p}$.

Theorem 3. Let $S_{n, h_{1}, h_{2}, \ldots, h_{n}}$ be a thorn star graph; then,

$$
\begin{aligned}
\mathrm{GC}\left(S_{n, h_{1}, h_{2}, \ldots, h_{n}}\right)= & (n-1)\left(n-2+h_{n}\right)\left(L+(n-2)\left(n-2+h_{n}\right)\right)+\sum_{i=1}^{n-1} h_{i}^{3}\left(h_{i}-1\right) \\
& +\left(n+h_{n}-2\right)^{2} h_{n}\left(h_{n}-1\right)+\left(n+h_{n}-2\right) h_{n}\left(2(n-1)\left(n-2+h_{n}\right)+L\right) \\
& +\left((3 n-5)\left(n+h_{n}-2\right)+2 L\right) \sum_{j=1}^{n-1} h_{j}^{2}+3\left(h_{n}+n-2\right) h_{n} \sum_{j=1}^{n-1} h_{j}^{2}+4 \sum_{1 \leq i<j \leq n-1} h_{i}^{2} h_{j}^{2} .
\end{aligned}
$$

Proof. The proof is followed by Theorem 1 .

Some special cases of Theorem 3 are discussed in the following corollaries.
Corollary 5. If thorn of length $h$ is attached with all the vertices other than the root vertex $h_{i}=h$, for $i \leq n-1$, then $L=(h n(n-1)) / 2$ and

$$
\begin{aligned}
\mathrm{GC}\left(\Gamma^{h}\right)= & (n-1)\left(n-2+h_{n}\right)\left(L+(n-2)\left(n-2+h_{n}\right)\right)+h^{3}(h-1)(n-1)+\left(n+h_{n}-2\right)^{2} h_{n}\left(h_{n}-1\right) \\
& +\left(n+h_{n}-2\right) h_{n}\left(2(n-1)\left(n-2+h_{n}\right)+L\right)+\left((3 n-5)\left(n+h_{n}-2\right)+h n(n-1)\right) \\
& \cdot \frac{h n(n-1)}{2}+3\left(h_{n}+n-2\right) h_{n} \frac{h n(n-1)}{2}+2 h^{4}(n-1)(n-2),
\end{aligned}
$$

where $\Gamma^{h}=S_{n, h, \ldots, h, h_{n}}$.

Corollary 6. If no thorn is attached with root vertex of thorn star, then

$$
\mathrm{GC}\left(\Gamma^{h}\right)=(n-1)(n-2)\left(L+(n-2)^{2}\right)+\sum_{i=1}^{n-1} h_{i}^{3}\left(h_{i}-1\right)+((3 n-5)(n-2)+2 L) \sum_{j=1}^{n-1} h_{j}^{2}+4 \sum_{1 \leq i<j \leq n-1} h_{i}^{2} h_{j}^{2}
$$

where $\Gamma^{h}=S_{n, h_{1}, h_{2} \cdots, h_{n-1}, 0}$.

Corollary 7. If no thorn is attached with root vertex and with other vertices a thorn of length $h$ is attached, then

$$
\begin{aligned}
\mathrm{GC}\left(\Gamma^{h}\right)= & (n-1)(n-2)\left(h(n-1)+(n-2)^{2}\right)+h^{3}(h-1)(n-1) \\
& +((3 n-5)(n-2)+2 h(n-1)) h^{2}(n-1)+2 h^{4}(n-1)(n-2),
\end{aligned}
$$


where $\Gamma^{h}=S_{n, h, \ldots, h, 0}$.

Now, we will discuss the GC index for the thorn ring graph.
Theorem 4. Let $C_{m, n}$ be a thorn graph of cycle graph $C_{n}$ with $n \geq 5$; then,

$$
\mathrm{GC}\left(C_{m, n}\right)= \begin{cases}\frac{(m-1)^{2} n\left(n^{2}-1\right)}{2}+(m-1)^{2}(m-2)(m-3) n \\ +(m-1)(m-2) n\left(\frac{n^{2}-1}{2}+2 n+\frac{(m-2)\left(n^{2}+4 n-1\right)}{2}\right) \\ +\frac{(m-1)^{2}(m-2)^{2} n(n-1)(n+9)}{8}, & \text { if } n \text { is odd, } \\ \frac{(m-1)^{2} n^{3}}{2}+(m-1)^{2}(m-2)(m-3) n & \\ +(m-1)(m-2) n\left(\frac{n^{2}}{2}+2 n+\frac{(m-2) n(n+4)}{2}\right) & \end{cases}
$$

Proof. The proof is followed by Theorem 1 .

\section{Conclusion}

In this section, we conclude our study as follows:

(i) Chemical applicability of GCI for several octane isomers is discussed, and it is found that it has high correlations with entropy, accentric factor, enthalpy of vaporization, standard enthalpy of vaporization, and standard heat of formation

(ii) The GC index of thorn graphs is obtained in its general form

(iii) The GC index of thorn paths, caterpillars, thorn rods, thorn stars, and thorn rings are also computed

(iv) A descriptor $M_{21}$ (sum of connection numbers of vertices of a graph) is provided in Lemma 1 that is called as connection degree sum

Now, we close this discussion that the various investigations are still needed for different (molecular) graphs or networks with the help of newly defined GC index.

\section{Data Availability}

The data used to support the findings of this study are cited at relevant places within article as references.

\section{Conflicts of Interest}

The authors declare that there are no conflicts of interest regarding this publication.

\section{References}

[1] F. M. Brückler, T. Došlić, A. Graovac, and I. Gutman, "On a class of distance-based molecular structure descriptors," Chemical Physics Letters, vol. 503, no. 4-6, pp. 336-338, 2011.

[2] H. Gonzalez-Diaz, S. Vilar, L. Santana, and E. Uriarte, "Medicinal chemistry and bioinformatics-current trends in drugs discovery with networks topological indices," Current Topics in Medicinal Chemistry, vol. 7, no. 10, pp. 1015-1029, 2007.

[3] R. Todeschini and V. Consonni, Molecular Descriptors for Chemoinformatics, Wiley-VCH Verlag GmbH, vol. 2, p. 1252, Weinheim, Germany, 2009.

[4] F. Yan, Q. Shang, S. Xia, Q. Wang, and P. Ma, "Application of topological index in predicting ionic liquids densities by the quantitative structure property relationship method," Journal of Chemical \& Engineering Data, vol. 60, no. 3, pp. 734-739, 2015.

[5] I. Gutman and N. Trinajstić, "Graph theory and molecular orbitals. Total $\varphi$-electron energy of alternant hydrocarbons," Chemical Physics Letters, vol. 17, no. 4, pp. 535-538, 1972.

[6] B. Furtula and I. Gutman, "A forgotten topological index," Journal of Mathematical Chemistry, vol. 53, no. 4, pp. 11841190, 2015. 
[7] S. Klavzar and I. Gutman, "Selected properties of the Schultz molecular topological index," The Journal for Chemical Information and Computer Scientists, vol. 36, pp. 1001-1003, 1996.

[8] A. Ali and N. Trinajstić, "A novel/old modification of the first Zagreb index," Molecular Informatics, vol. 37, no. 6-7, Article ID 1800008, 2018.

[9] U. Ali, M. Javaid, and A. Kashif, "Modified Zagreb connection indices of the T-sum graphs," Main Group Metal Chemistry, vol. 43 , no. 1 , pp. $43-55,2020$.

[10] M. Javaid, U. Ali, and M. K. Siddique, "Novel connection based Zagreb indices of several wheal-related graphs," Computational Journal of Combinatorial Mathematics, vol. 2, pp. 31-58, 2020.

[11] A. Ahmad, M. F. Nadeem, K. Elahi, and R. Hasni, "Computing topological indices of chemical structures of the conductive 2D MOFs," Journal of Information and Optimization Sciences, vol. 42, no. 3, pp. 563-578, 2021.

[12] A. Ahmad, M. A. Asim, and M. F. Nadeem, "Polynomials of degree-based indices of metal-organic networks," Combinatorial Chemistry and High Throughput Screening, vol. 24, pp. 1-9, 2020.

[13] M. Siddique, Y. Chu, M. Nasir, M. Nadeem, and M. Hanif, "On topological descriptors of ceria oxide and their applications," Main Group Metal Chemistry, vol. 44, no. 1, pp. 103-116, 2021.

[14] H. Wiener, "Structural determination of paraffin boiling points," Journal of the American Chemical Society, vol. 69, no. 1, pp. 17-20, 1947.

[15] I. Gutman, "Selected properties of the Schultz molecular topological index," Journal of Chemical Information and Computer Sciences, vol. 34, no. 5, pp. 1087-1089, 1994.

[16] I. Gutman, "Distance in thorny graph," Publications de l'Institut Mathématique (Beograd), vol. 63, pp. 31-36, 1998.

[17] L. Bytautas, D. Bonchev, and D. J. Klein, "On the generation of mean Wiener numbers of thorny graphs," MATCH Communications in Mathematical and in Computer Chemistry, vol. 44, pp. 31-40, 2001.

[18] B. Zhou, "On modified Wiener indices of thorn trees," Kragujevac Journal of Mathematics, vol. 27, pp. 5-9, 2005.

[19] S. Li, "Zagreb polynomials of thorn graphs," Kragujevac Journal of Science, vol. 33, pp. 33-38, 2011.

[20] I. Gutman, B. Furtula, J. Tovsic, E. Mohamed, and M. El marraki, "On terminal Wiener indices of kenograms and plerograms," Iranian Journal of Mathematical Chemistry, vol. 4, no. 1, pp. 77-89, 2013.

[21] Y. Alizadeh, A. Iranmanesh, T. Došlić, and M. Azari, "The edge wiener index of suspensions, bottlenecks, and thorny graphs," Glasnik Matematicki, vol. 49, no. 1, pp. 1-12, 2014.

[22] M. Azari and A. Iranmanesh, "Dendrimer graphs as thorn graphs and their topological edge properties," National Academy Science Letters, vol. 39, no. 6, pp. 455-460, 2016.

[23] D. Bonchev and D. J. Klein, "On the Wiener number of thorn trees, stars, rings and rods," Croatica Chemica Acta, vol. 75, no. 2, pp. 613-620, 2002.

[24] M. Azari, "On the Gutman index of Thorn graphs," Kragujevac Journal of Science, vol. 40, no. 40, pp. 33-48, 2018. 